\title{
Clinical Efficacy and Cost Analysis of Antibiotics for Treatment of Uncomplicated Urinary Tract Infections in the Emergency Department of a Tertiary Hospital in Saudi Arabia
}

\author{
Menyfah Q Alanazi $\mathbb{D}^{1-3}$ \\ 'Drug Policy and Economics Center, King \\ Abdulaziz Medical City, Ministry of \\ National Guard-Health Affairs, Riyadh, \\ Saudi Arabia; ${ }^{2}$ King Abdullah \\ International Medical Research Center, \\ Riyadh, Saudi Arabia; ${ }^{3}$ King Saud Bin \\ Abdulaziz University for Health Sciences, \\ Riyadh, Saudi Arabia
}

Purpose: Uncomplicated urinary tract infections (uUTIs) are one of the main reasons for emergency department (ED) visits. Many antibiotics can be used for uUTI treatment. Currently, no data concerning uUTIs and cost-effectiveness have been reported in Saudi Arabia. This study aimed to investigate antibiotic and cost-effectiveness of beta-lactams, fluoroquinolones, and nitrofurantoin as first-line uUTI treatment.

Patients and Methods: This study was a retrospective cohort based on a five-arm comparative outcome analysis. A cost-effectiveness analysis and comparative group of uUTI treatments in the ED at King Abdulaziz Medical City (KAMC) in Saudi Arabia over a three-month follow-up period was done. The patient group consisted of those presenting to the ED with uUTIs who were treated initially with one of five antibiotics: (1) amoxicillin/clavulanic acid, (2) cefuroxime, (3) ciprofloxacin, (4) nitrofurantoin, or (5) norfloxacin. The main outcomes were effectiveness in terms of cure rates, symptom-free days (SFDs), and estimations of cost-effectiveness among this group.

Results: A total of 865 adult patients who presented with uUTIs were enrolled. Most patients $(89.5 \%)$ completely recovered, whereas $10.5 \%$ of patients were readmitted to the ED with recurrent infections. Effectiveness in terms of the highest cure rate was observed with nitrofurantoin and amoxicillin/clavulanic acid (93.2\% and 92.2\%, respectively) followed by norfloxacin, cefuroxime, and ciprofloxacin; no significant differences in cure rates were found among these antibiotics. Antibiotic effectiveness in terms of SFDs showed that nitrofurantoin produced the longest SFD period ( 76 days) followed by amoxicillin/clavulanic acid (69 days). A cost-effectiveness analysis in terms of uUTI cure rates and number of SFDs indicated that nitrofurantoin presented the highest cost-effectiveness followed by amoxicillin/ clavulanic acid, norfloxacin, ciprofloxacin, and cefuroxime.

Conclusion: A comparison of five antibiotics for uUTI treatment did not yield clinically significant differences in cure rates. Nitrofurantoin was more cost-effective than the other antibiotics.

Keywords: urinary tract infection, antibiotics, cost effectiveness, cure rate

\section{Introduction}

Urinary tract infections (UTIs) are a serious worldwide health problem affecting all age groups and both genders. ${ }^{1-3}$ Urinary tract infections are the third most common types of infections worldwide, affecting both outpatients and inpatients. ${ }^{1}$ An uncomplicated urinary tract infection (UUTI) is among the most common infections in emergency departments. ${ }^{4-6}$ Escherichia coli is the most common cause of
Drug Policy and Economics Center, King Abdulaziz Medical City, Ministry of National Guard-Health Affairs, P.O. Box 22390

Riyadh, I I 426, Saudi Arabia

Tel +966 I I-80 I I I I

Email menefah@gmail.com 
uncomplicated UTI. ${ }^{1,6-8}$ Most uncomplicated urinary tract infections occur in young women, with far fewer cases occurring in men. ${ }^{8,9}$

In Saudi Arabia, UTI is the second most common infection that prompts emergency department (ED) visits. In addition, the prevalence of UTIs among adult patients was found to be $14.6 \%$ of all ED visits. ${ }^{2,10}$

UTIs have a significant impact on patient health-related quality of life (HRQoL) and costs. The mean baseline HRQoL of Saudi women with UTI was $72 \%$ with varying effects depending on the socio-demographic characteristics and other comorbid chronic diseases. ${ }^{2,10,11}$ The mean cost for UTI treatment in EDs in Saudi Arabia is estimated as $\$ 134.56 \pm \$ 31.34$ (United States [US] dollars). ${ }^{2}$

UTI is among the most common reasons for outpatient visits and antibiotic use in adult populations. Antibiotics are the cornerstone for UTI treatment, and several types of antibiotics are currently recommended for the treatment of uUTIs; these antibiotics include beta lactams, fluoroquinolones, nitrofurantoin, and sulfamethoxazole/trimethoprim. ${ }^{2-6,12,13}$

The use of effective antibiotics for the treatment of uUTI is necessary to improve patient HRQoL and reduce the risk of progression to a more serious condition. Extensive and inappropriate antibiotic use can have a negative impact, including treatment failure, antibiotic resistance, risk of complications, and increased cost of treatment, on patients and society. ${ }^{1,2,7,11,12,14-16}$

The increasing prevalence of antibacterial resistance among community uro-pathogens affects the diagnosis and management of this clinical syndrome. ${ }^{3,6}$

The challenges of managing UTIs in an ED include limited medical history, lack of follow-up, and lack of culture and susceptibility results. Most patients do not require an extensive diagnostic evaluation and can be treated as outpatients using administration of oral antibiotics. 5,6

To date, no published studies have compared relative cost-effectiveness of beta lactams, fluoroquinolones, and nitrofurantoin for treatment of uUTIs in Saudi Arabia, so minimal information to guide clinicians with respect to their prescribing choices based on the cost effectiveness of antibiotics exists.

In Saudi Arabia, the government provides free healthcare services to all citizens through a number of government agencies; however, this policy has generated cost pressures on the government, especially in view of the rapid population growth.

The objective of this study was to investigate the effectiveness and cost impact of using beta lactams, fluoroquinolones, and nitrofurantoin for first-line uUTI treatment in Saudi Arabia.

This study will provide new information on the costeffectiveness and budget impact of oral antibiotics for uUTI treatment: (1) beta lactams (amoxicillin/clavulanic acid [Augmentin], cefuroxime), (2) fluoroquinolones (ciprofloxacin, norfloxacin), and (3) nitrofurantoin for uUTI treatment and a comparison of efficacy and direct costs.

\section{Methods}

\section{Study Setting}

This study represents a real-world clinical setting in the ED of King Abdulaziz Medical City (KAMC). KAMC is a university-affiliated tertiary care center in Riyadh, Saudi Arabia, which has been accredited by the Joint Commission International.

\section{Study Design}

This study was a retrospective cohort based on a five-arm comparative outcome analysis. A cost effectiveness analysis in conjunction with comparative antibiotic treatment of uUTIs in the ED at KAMC in Saudi Arabia with a three-month follow-up period was done. Decision trees were used to compare the costs and effects of different antibiotics (amoxicillin/ clavulanic acid, cefuroxime, ciprofloxacin, nitrofurantoin, and norfloxacin) to treat uUTIs in patients who visited the ED (Figure 1).

\section{Study Population}

The population consisted of adults $>18$ years old who presented to the ED with symptoms indicating uUTI as diagnosed by emergency physicians and who were given one of five antibiotics as first-line treatment: (1) amoxicillin/clavulanic acid, (2) cefuroxime, (3) ciprofloxacin, (4) nitrofurantoin, or (5) norfloxacin after which the patients were then followed up to measure the efficacy and analysis of the impact cost of treatment. The usual criteria applied to an initial UTI diagnosis consist of medical history, clinical findings, and results from a dipstick test.

Any cases with medical histories of diabetes, renal failure, UTI with sepsis or bacteremia, catheterassociated UTI, and/or comorbidities were excluded.

\section{Data Collection}

Several sets of data were reviewed. Patient characteristics included demographic data, number of visits to the ED for 


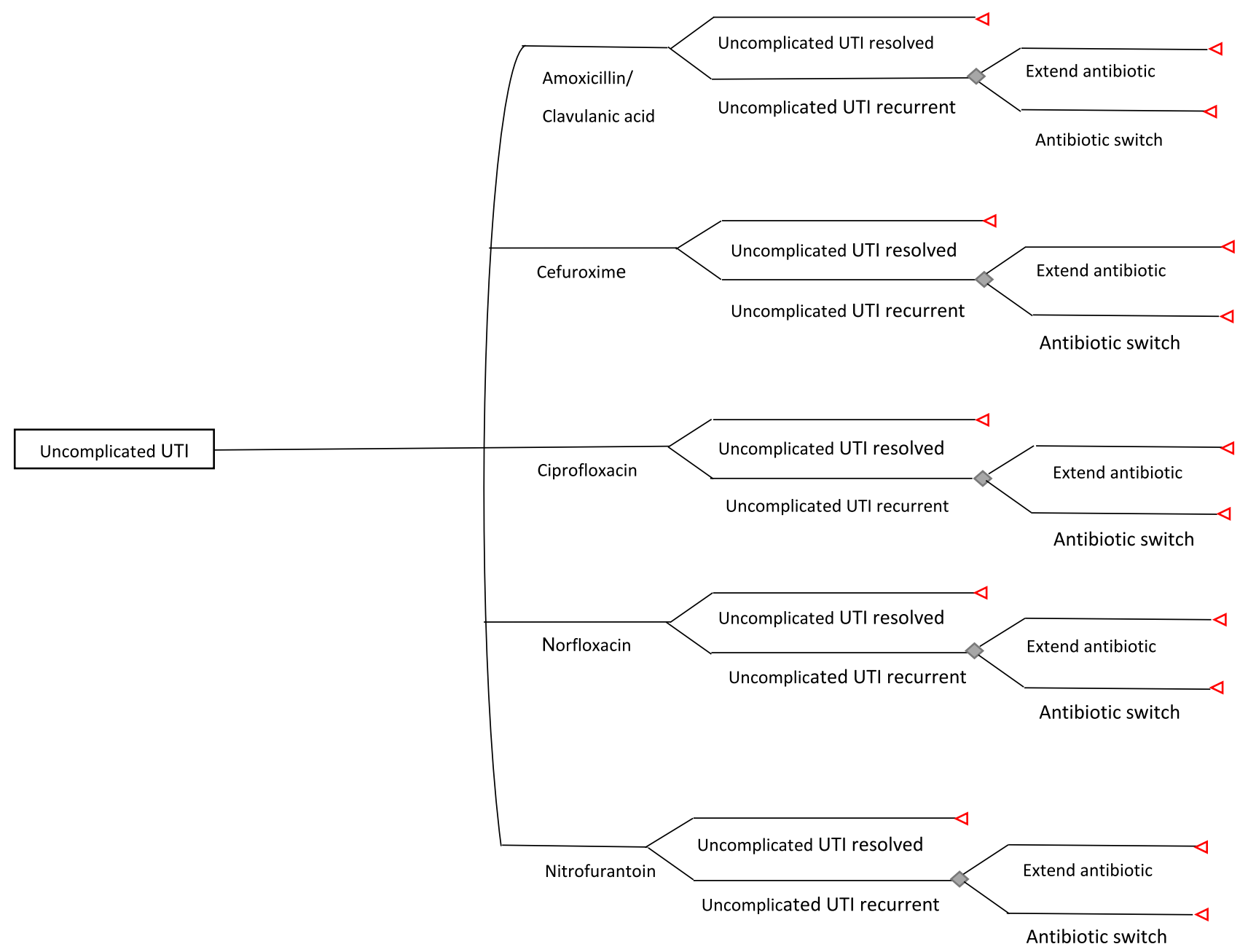

Figure I Antibiotic decision tree for cost-effectiveness analysis of uncomplicated urinary tract.

each patient within three months, and recurrent episodes of UTI. The main outcome characteristics included antibiotic and cost-effectiveness of the five antibiotics used for treating uUTIs.

The effectiveness of these antibiotics is defined as complete recovery from a uUTI during study period. The measurement of treatment effectiveness in terms of clinical cure and of number of symptom-free days (SFDs) was evaluated. The clinical cure rate was measured as the percentage of patients who achieved resolution of uUTI symptoms and had no recurrent episodes during the study period after being treated with the initial antibiotic (no others were needed).

The number of SFDs was defined as the number of symptom-free days due to a uUTI during the study period after treatment with the initial antibiotic.

For the cost-effectiveness analysis, the costeffectiveness ratio in terms of clinical cure was defined as cost of initial antibiotic during the study period divided by proportion of clinical cure (Cost/Cure).

The cost-effectiveness ratio in terms of SFDs was defined as total cost of initial antibiotic during the study period divided by number of SFDs (Cost/ SFD).

The incremental cost-effectiveness ratio (ICER) was calculated for each of the five antibiotics used in the study treatment of the uUTI, and the difference between the cost divided by difference between the cure was determined.

Estimated prices of antibiotics for the treatment of uUTI were computed based on prices of the medicines from the Saudi Food and Drug Administration (FDA). US dollars were used to calculate the costs associated with the study. ${ }^{17}$

\section{Ethical Issues}

This study was approved by the research committee at King Abdullah International Medical Research Center 
(KAIMRC), King Saud Bin-Abdulaziz University for Health Sciences, Riyadh, Saudi Arabia (RC20/551/R). An informed consent to review medical files was not required and waived by the research committee at KAIMRC, as the study was a retrospective chart review.

This study followed the recommendations of the International Conference on Harmonization for Good Clinical Practice (ICH-GCP) and in compliance with the Declaration of Helsinki.

Patient confidentiality and privacy of information were secured by the principal investigator.

\section{Data Management and Analysis}

The SPSS statistical software (version 22; IBM, Armonk, NY, USA) was used for data entry and analysis. Bivariate analysis using Pearson's $\chi^{2}$ test was used for the effectiveness of an antibiotic. For cost analysis, data were summarized in the form of mean \pm standard deviation (SD) for continuous variables and numbers and percentages for categorical variables, and P-values $<0.05$ were considered statistically significant.

\section{Results}

During the study period, a total of 876 patients (age $>18$ ) were diagnosed with uUTI and enrolled in the study; 784 patients demonstrated a complete recovery from a single visit (representing 89.5\% of the study patients), whereas 92 patients were readmitted to the ED with same, recurring uUTI (representing $10.5 \%$ of the study patients). Female patients made up most patients $(65.7 \%)$ visiting the ED with a uUTI. The male to female ratio was $1: 1.8$ with mean patient age of $37.3 \pm 11.65$ years and range 19-64 years.

Most patients received one antibiotic during the study period ( $89.5 \%$ of patients), $8.56 \%$ received two courses of antibiotics, and $1.6 \%$ of patients received three courses during the study period.

\section{Clinical Outcomes \\ Effectiveness}

The measurement of treatment effectiveness was done in terms of cure rate and number of SFDs.

Figure 2 illustrates slight differences in cure rates among the five antibiotics. Slight differences in recurrent infection rates were also noticed among the five target antibiotics.

Table 1 shows the highest cure rate occurred after nitrofurantoin and amoxicillin/clavulanic acid administration (93.2\% and 92.2\%, respectively) followed by norfloxacin, cefuroxime, and ciprofloxacin $(89.9 \%, 88.2 \%$, and $86.4 \%$, respectively). No significant differences among cure rates of antibiotics were found $(p=0.399)$. The least number of recurrent infections occurred in patients treated with nitrofurantoin $(6.8 \%)$ followed by amoxicillin/clavulanic acid (7.8\%) and then norfloxacin (10.2\%). No clinically significant differences among recurrent rates of infection among antibiotics were found $(\mathrm{p}=0.431)$.

The measurement of treatment effectiveness in terms of the number of SFDs showed nitrofurantoin produced 76

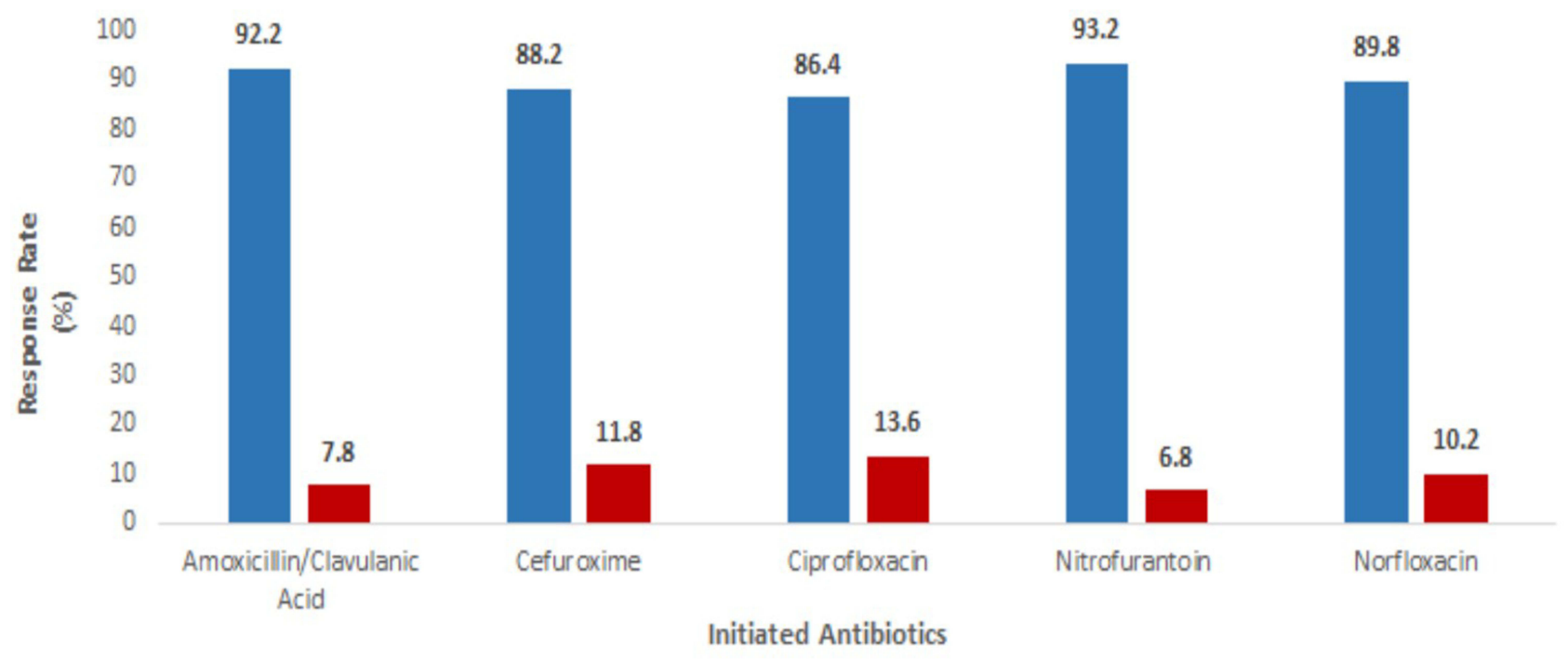

- Cure Rate

- Recurrent Infection Rate

Figure 2 Cure rate (\%) and recurrent infection rate (\%) for different antibiotics. 
Table I Outcome Treatment of Uncomplicated Urinary Tract Infection (uUTI) Among Different Antibiotics During Study Period According to Cure Rate

\begin{tabular}{|c|c|c|c|c|c|c|c|c|c|}
\hline \multirow[t]{3}{*}{ Initiated Antibiotic } & \multirow{3}{*}{$\begin{array}{l}\text { Sample, } \\
N(\%) \\
(N=876)\end{array}$} & \multicolumn{4}{|c|}{ Completely Recovery } & \multicolumn{4}{|c|}{ Recurrent Infection } \\
\hline & & \multirow{2}{*}{$\begin{array}{l}\text { Cure } \\
\text { rates }^{a} \\
(\mathrm{~N}, \%)\end{array}$} & \multicolumn{3}{|c|}{ Cost } & \multirow{2}{*}{$\begin{array}{c}\text { Recurrence } \\
\text { Infection Rates } \\
\text { (N, \%) }\end{array}$} & \multicolumn{3}{|c|}{ Cost } \\
\hline & & & $\begin{array}{c}\text { Mean } \pm \\
\text { SD }\end{array}$ & $95 \% \mathrm{Cl}$ & P value & & $\begin{array}{c}\text { Mean } \pm \text { SD } \\
(\$)\end{array}$ & $95 \% \mathrm{Cl}$ & $P$ value \\
\hline Amoxicillin/clavulanic acid & 153 (17.47) & $|4|(92.2)$ & $25.72 \pm 12.57$ & $23.62-27.81$ & $<0.0001$ & $12(7.8)$ & $54.11 \pm 29.15$ & $35.59-72.62$ & $<0.0001$ \\
\hline Cefuroxime & $389(44.41)$ & 343 (88.2) & $33.91 \pm 9.77$ & $32.87-34.94$ & & $46(11.8)$ & $66.96 \pm 23.98$ & $59.84-74.1$ & \\
\hline Ciprofloxacin & $59(6.74)$ & $51(86.4)$ & $19.67 \pm 14.97$ & $15.46-23.89$ & & $8(13.6)$ & $35.84 \pm 19.14$ & $\mid 9.83-61.84$ & \\
\hline Nitrofurantoin & $59(6.74)$ & $55(93.2)$ & $20.67 \pm 8.88$ & $\mid 8.27-23.1$ & & $4(6.8)$ & $47.69 \pm 25.73$ & $6.76-88.63$ & \\
\hline Norfloxacin & $216(24.66)$ & $194(89.8)$ & $19.55 \pm 13.50$ & $|7.64-2| .45$ & & $22(10.2)$ & $47.94 \pm 28.50$ & $35.3-60.58$ & \\
\hline Total & 876 & $784(89.5)$ & $26.29 \pm 13.35$ & $25.40-27.19$ & & $92(10.5)$ & $56.28 \pm 27.26$ & $50.78-61.77$ & \\
\hline
\end{tabular}

Notes: ${ }^{a}$ No clinically significant differences between six different antibiotics were found; $p=0.399$.

Abbreviations: SD, standard deviation; $\mathrm{Cl}$, confidence interval.

Table 2 Costs, Cure, and Cost-Effectiveness Ratios of a Set of Interrelated Antibiotics

\begin{tabular}{|c|c|c|c|c|c|}
\hline & $\begin{array}{c}\text { Amoxicillin/ } \\
\text { Clavulanic Acid }\end{array}$ & Cefuroxime & Ciprofloxacin & Norfloxacin & Nitrofurantoin \\
\hline \multicolumn{6}{|l|}{ Method I: Cost consequence analysis } \\
\hline Cost per course & $\$ 12.00$ & $\$ 34.65$ & $\$ 21.73$ & $\$ 12.31$ & $\$ 3.38$ \\
\hline Cost per three months & $\$ 10,608.00$ & $\$ 29,279.25$ & $\$ 17,992.44$ & $\$ 10,598.91$ & $\$ 3,018.34$ \\
\hline \multicolumn{6}{|l|}{ Outcome } \\
\hline Cured\% & 92.2 & 88.2 & 86.4 & 89.9 & 93.2 \\
\hline Symptoms free days (SFD) in three months (days) & 69 & 60 & 69 & 60 & 76 \\
\hline \multicolumn{6}{|l|}{ Number recurrent episodes } \\
\hline One & $10(6.5)$ & $39(10)$ & $6(10.2)$ & $17(7.9)$ & $3(6.8)$ \\
\hline Two & $2(1.3)$ & $6(1.5)$ & $2(3.4)$ & $4(1.9)$ & 0 \\
\hline Three & 0 & $\mathrm{I}(0.3)$ & 0 & $\mathrm{I}(0.5)$ & 0 \\
\hline \multicolumn{6}{|l|}{ Method 2: Cost-effectiveness ratio: (CE Ratio) } \\
\hline Cure & $\$ 11,505.42$ & $\$ 33,196.43$ & $\$ 20,353.44$ & $\$ 11,789.67$ & $\$ 3,238.56$ \\
\hline SFD & $\$ 153.74$ & $\$ 487.99$ & $\$ 260.76$ & $\$ 176.65$ & $\$ 39.72$ \\
\hline
\end{tabular}

Notes: Cure is the cost of antibiotics divided by the cure rate (Cost/Cure), SFD ratio is a cost of antibiotics divided by symptom-free days (Cost/ SFD). Abbreviation: SFD, Symptom-free days.

SFDs followed by amoxicillin/clavulanic acid (69 days) treatment, and no differences between cefuroxime, ciprofloxacin, and norfloxacin were detected, as shown in Table 2.

The number of frequent uUTI episodes were lower with nitrofurantoin (one episode) followed by amoxicillin/clavulanic acid (two episodes) and then cefuroxime, ciprofloxacin, and norfloxacin with three episodes each reported during the study period (Table 2).

\section{Cost Analysis}

Table 2 illustrates the comparison between costeffectiveness ratios of five antibiotics.
Cost-effectiveness was calculated in terms of cure and SFD ratios. The findings indicate that initiation of nitrofurantoin for uUTI management presented a higher cost-effectiveness followed by amoxicillin/clavulanic acid, norfloxacin, ciprofloxacin, and finally cefuroxime $(\$ 3,238.56, \$ 11,505.42$, $\$ 11,789.67, \$ 20,353.44$, and $\$ 33,196.43$, respectively).

Figure 3 indicates that nitrofurantoin was more effective and cost less, and no differences between the costeffectiveness of amoxicillin/clavulanic acid and norfloxacin were found. Cefuroxime had the lowest cost-effectiveness. The cost-effectiveness of nitrofurantoin initiation was 


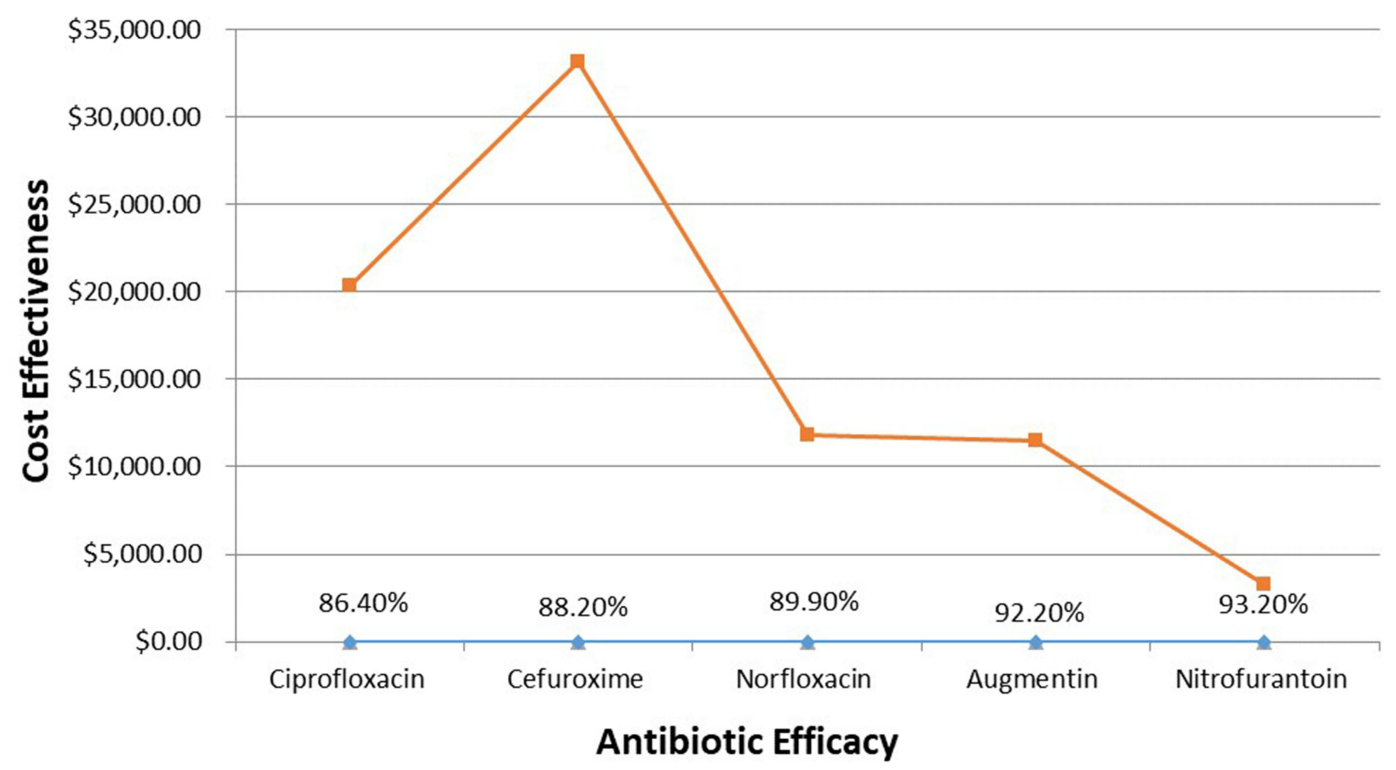

Figure 3 Effectiveness and cost effectiveness of study antibiotics.

approximately triple the cost-effectiveness of norfloxacin and augment, six times that of ciprofloxacin, and ten times that of cefuroxime.

Cost-effectiveness in terms of SFD ratio showed nitrofurantoin initiation for the management of uUTI had higher cost-effectiveness than other antibiotics in all analyses followed by amoxicillin/clavulanic acid, norfloxacin, ciprofloxacin, and cefuroxime (\$39.72, \$153.74, \$176.65, $\$ 260.76$, and \$487.99, respectively).

The ICER was calculated for each of the five antibiotics used in the treatment of the uUTI, and results are given in Table 3 . The results demonstrate that nitrofurantoin had less ICER when compared with other oral antibiotics, which represented more effectiveness and less cost, expressed as more cost-effectiveness.

Negative results indicate cost savings. Negative ICER score for antibiotics indicate that these antibiotics are the most effective with a corresponding reduction in costs and are dominant alternatives over other antibiotics used for the treatments.

\section{Discussion}

The present study was the first cost-effectiveness study conducted in Saudi Arabia that used real-world cost data to compare five oral antibiotics for the management of uUTIs in the ED: (1) amoxicillin/clavulanic acid, (2) cefuroxime, (3) ciprofloxacin, (4) norfloxacin, and (5) nitrofurantoin. Use of a cost-effectiveness analysis to estimate the economic and health implications of five oral antibiotics for treatment of UTI proved to be an effective method for examining these two parameters.

Fluoroquinolones, sulfonamides, amoxicillin/clavulanic, and nitrofurantoin were found to be the most frequently used antibiotics for uUTI treatment. Limited evidence on the cost-effectiveness of antibiotics for urinary tract infection in ED exists.

Table 3 Comparison Between Different Incremental Cost Effectiveness Ratios (ICER) of Antibiotics During the Study Period

\begin{tabular}{|c|c|c|c|c|c|}
\hline \multirow[b]{2}{*}{ Scientific Name } & \multicolumn{5}{|c|}{ Incremental Cost Effectiveness Ratio (ICER) } \\
\hline & Amoxicillin/Clavulanic Acid & Cefuroxime & Ciprofloxacin & Norfloxacin & Nitrofurantoin \\
\hline Amoxicillin/clavulanic acid & $\$ 0.00$ & $-\$ 487.33$ & $-\$ 132.87$ & $\$ 0.00$ & $-\$ 792.00$ \\
\hline Cefuroxime & $-\$ 487.33$ & $\$ 0.00$ & $\$ 654.81$ & $-\$ 1,146.67$ & $-\$ 548.27$ \\
\hline Ciprofloxacin & $-\$ 132.87$ & $\$ 654.81$ & $\$ 0.00$ & $-\$ 220.19$ & $-\$ 229.80$ \\
\hline Norfloxacin & $\$ 0.00$ & $-\$ 1,146.67$ & $-\$ 220.19$ & $\$ 0.00$ & $-\$ 240.00$ \\
\hline Nitrofurantoin & $-\$ 792.00$ & $-\$ 548.27$ & $-\$ 229.80$ & $-\$ 240.00$ & $\$ 0.00$ \\
\hline
\end{tabular}


In the current study, the highest cure rates occurred with nitrofurantoin and amoxicillin/clavulanic acid, which were slightly higher than norfloxacin, cefuroxime, and ciprofloxacin, with no clinically significant differences between cure rates of five antibiotics. This finding was consistent with cure rates determined in a study conducted in the UK in which no significance differences between ciprofloxacin, nitrofurantoin, norfloxacin, cephalosporin, and trimethoprim for the treatment of uUTI in young women were found. ${ }^{18}$ Other studies reported that ciprofloxacin was more clinically curative than amoxicillin/clavulanic, whereas in terms of adverse effects, no significant differences were detected. ${ }^{19,20}$

The present study showed the least recurrent infection was observed after treatment with nitrofurantoin followed by amoxicillin/clavulanic acid and norfloxacin. These results contradict those from other studies in which it was demonstrated that ciprofloxacin, cephalexin, and coamoxiclav were associated with lower rates of treatment failure and similar risks of UTI-related hospitalizations or death in older people than was nitrofurantoin. ${ }^{21}$

Information available in Saudi Arabia concerning the differences in cure rates based on sensitivity found nitrofurantoin had the highest cure rate followed by ciprofloxacin, amoxicillin/clavulanic acid, and norfloxacin and the least sensitive was reported as co-trimoxazole out of all of the tested antibiotics. ${ }^{7}$

This finding was consistent with other studies that reported nitrofurantoin presented the lowest resistance and trimethoprim-sulfamethoxazole produced the highest. ${ }^{13,22}$

Resistance development in common UTI pathogens (especially to fosfomycin, sulfamethoxazole-trimethoprim, fluoroquinolones, and third-generation cephalosporins), have seriously restricted therapeutic options, especially in outpatient settings. ${ }^{22}$

Ciprofloxacin is an important antibiotic for treatment of more serious infections, and overuse of this antibiotic may have serious implications for the treatment of more serious infections. The Infectious Diseases Society of America (IDSA) guidelines have reduced fluoroquinolones from first-line therapy to alternative agents for uUTI treatment. $^{23}$

In the present study, the results indicate that initiation of nitrofurantoin for the management of uUTI showed a lower ICER and higher cost-effectiveness followed by amoxicillin/clavulanic acid, norfloxacin, and ciprofloxacin.
Moreover, cefuroxime had a higher ICER than other oral antibiotics in all analyses.

The ICER of nitrofurantoin was approximately $\$ 3,238$. Amoxicillin/clavulanic acid and norfloxacin versus nitrofurantoin had triple the ICER, ciprofloxacin was six times the ICER of nitrofurantoin, and cefuroxime was 10 times the ICER of nitrofurantoin. Amoxicillin/clavulanic acid and norfloxacin were similar in cost-effectiveness in terms of cure ratios but less cost-effective in terms of SFDs. No other local studies to compare relative costeffectiveness of these antibiotics in Saudi Arabia have been published. When compared with previous studies in other countries, it was found that trimethoprim was reported to be a more cost-effective treatment than nitrofurantoin and fosfomycin when resistance level to trimethoprim was $<30 \%$, but at resistance levels of $\geq 35 \%$ for trimethoprim, both fosfomycin and nitrofurantoin were shown to be more cost-effective. ${ }^{24}$ In other studies, the sulfonamides, fluoroquinolones, and nitrofurantoin were reported to have similar costs per patient. ${ }^{25}$

In study conducted in the US, it was found that nitrofurantoin for the treatment of uUTIs minimized costs when compared with trimethoprim-sulfamethoxazole in many areas of the US and was cost-competitive when compared with generic ciprofloxacin. ${ }^{26}$

Despite the lower cost of and less resistance presented by nitrofurantoin, it is still less commonly used for the treatment of uUTIs.

Although a limited number of studies have evaluated the effectiveness of antibiotics for the treatment of uUTI, differences in cure rates and cost-effectiveness among countries can be found. Local sensitivities and antibiotic prices are important when calculating cost-effectiveness and making suitable choices.

Sensitivity and resistance play an important role when considering the cost-effectiveness of antibiotics; when the sensitivity increases, the efficacy of the treatment increases, whereas when resistance increases, the efficacy decreases, and the cost of UTI treatment will increase. Overall, the cost-effectiveness of antibiotics differs from country to country due to variations in local sensitivity, cost of antibiotics, and clinical practice guidelines.

The results of this study may help guide clinicians when choosing the most cost-effective antibiotic for the treatment of uUTI based on the local knowledge of resistance levels. Treatment of an uncomplicated uUTI is often acute for which good treatment decisions must be made rapidly and promptly in order to prevent complications. 


\section{Limitations}

This study has several limitations. First, the study was conducted in only one setting; thus, this ED does not represent other healthcare settings. Second, the cost of antibiotics differs from one manufacturer to another. The antibiotic costs included in my analyses reflect the trade name of antibiotics used during study period, which may differ in other settings in Saudi Arabia; therefore, the findings cannot be generalized to the entire country. Finally, other limitations may have resulted in over- or under-estimations of the actual costs associated with UTIs. Large-scale prospective studies are recommended to help yield a more accurate determination of the costeffectiveness of UTI treatments in Saudi Arabia.

\section{Conclusion}

All five antibiotics in the current study proved to be effective for the treatment of uUTI. From a costeffectiveness point of view, nitrofurantoin was more costeffective than the four other studied antibiotics that were used for treatment of uUTI. It was also found that amoxicillin/clavulanic acid produced similar cost-effectiveness compared with norfloxacin in terms of cure rates but less in term of SFDs. Results of this study cannot be readily generalized to other countries since regional differences in antibiotic resistance patterns and costs exist.

Additional studies should be performed in terms of cost-effective uUTI treatments in Saudi Arabia.

\section{Acknowledgments}

The author would like to acknowledge the King Abdullah International Medical Research Center (KAIMRC) for their support. Special thanks go to the KAIMRC Publication Office, Dr. Ahmed Alaskar, Dr. Ghiath Hassan Ahmad, Fares AlShwiman, Haneen Al-Zahrani, Hana Alabdulkareem, Dr. Falwah Alqahatani, and Dr. Hajer Alqahani.

\section{Disclosure}

The author reports no conflicts of interest in this work.

\section{References}

1. Gajdács M. The importance of reporting clinical and epidemiological data in urology: local experiences and insights from the international literature. Medicina. 2020;56(11):581. PMID: 33143077; PMCID: PMC7693886. (no 1). doi:10.3390/medicina56110581

2. Alanazi MQ. An evaluation of community-acquired urinary tract infection and appropriateness of treatment in an emergency department in Saudi Arabia. Ther Clin Risk Manag. 2018;14:2363-2373. doi:10.2147/TCRM.S178855
3. Gajdács M, Ábrók M, Lázár A, Burián K. Comparative epidemiology and resistance trends of common urinary pathogens in a tertiary-care hospital: a 10-year surveillance study. Medicina. 2019;55(7):356. PMID: 31324035; PMCID: PMC6681214. doi:10.3390/medicina55070356

4. Gajdács M, Ábrók M, Lázár A, Burián K. Epidemiology and antibiotic resistance profile of bacterial uropathogens in male patients: a 10-year retrospective study. Farmacia. 2021;69(3):530-539. ISSN 0014-8237 (print); 2065-0019 (online). doi:10.31925/farmacia.2021.3.16

5. Long B, Koyfman A. The emergency department diagnosis and management of urinary tract infection. Emerg Med Clin North Am. 2018;36 (4):685-710. PMID: 30296999. doi:10.1016/j.emc.2018.06.003

6. Takhar SS, Moran GJ. Diagnosis and management of urinary tract infection in the emergency department and outpatient settings. Infect Dis Clin North Am. 2014;28(1):33-48. PMID: 24484573. doi:10.1016/j.idc.2013.10.003

7. Alanazi MQ, Alqahtani FY, Aleanizy FS. An evaluation of E. coli in urinary tract infection in emergency department at KAMC in Riyadh, Saudi Arabia: retrospective study. Ann Clin Microbiol Antimicrob. 2018;17:3. doi:10.1186/s12941-018-0255-z

8. Mehnert-Kay SA. Diagnosis and management of uncomplicated urinary tract infections. Am Fam Physician. 2005;72:451-456. PMID: 16100859.

9. Farrell K, Tandan M, Hernandez Santiago V, et al. Treatment of uncomplicated UTI in males: a systematic review of the literature. BJGP Open. 2021;5(2):bjgpopen20X101140. doi:10.3399/bjgpopen20X101140

10. Alanazi MQ, Salam M, Alqahtani FY, et al. An evaluation of antibiotics prescribing patterns in the emergency department of a tertiary care hospital in Saudi Arabia. Infect Drug Resist. 2019;12:3241-3247. doi:10.2147/IDR.S211673

11. Alanazi MQ. Evaluation of health-related quality of life in women with community-acquired urinary Tract infections using the EQ-5D3L in Saudi Arabia. Patient Prefer Adherence. 2020;14:2419-2426. doi:10.2147/PPA.S277367

12. Sanyal C, Husereau DR, Beahm NP, et al. Cost-effectiveness and budget impact of the management of uncomplicated urinary tract infection by community pharmacists. BMC Health Serv Res. 2019;19(1):499. doi:10.1186/s12913-019-4303-y

13. Gajdács M, Bátori Z, Ábrók M, et al. Characterization of resistance in gram-negative urinary isolates using Existing and novel indicators of clinical relevance: a 10-year data analysis. Life. 2020;10(2):16. PMID: 32054054; PMCID: PMC7175163. doi:10.3390/life10020016

14. Alanazi MQ, Al-Jeraisy MI, Salam M. Prevalence and predictors of antibiotic prescription errors in an emergency department, Central Saudi Arabia. Drug Health Patient Saf. 2015;7:103-111.

15. Bermingham SL, Ashe JF. Systematic review of the impact of urinary tract infections on health-related quality of life. BJU Int. 2012;110 (11c):E830-E836. doi:10.1111/j.1464-410X.2012.11337.x

16. Le TP, Miller LG. Empirical therapy for uncomplicated urinary tract infections in an era of increasing antimicrobial resistance: a decision and cost analysis. Clin Infect Dis. 2001;33(5):615-621. doi:10.1086/322603

17. Saudi Food \& Drug Authority. Drugs List. Available from: https://www. sfda.gov.sa/index.php/en/drugs-list. Accessed November 17, 2021.

18. Lawrenson RA, Logie JW. Antibiotic failure in the treatment of urinary tract infections in young women. J Antimicrob Chemo. 2001;48(6):895-901. doi:10.1093/jac/48.6.895

19. Hooton TM, Scholes D, Gupta K, et al. Amoxicillin-clavulanate vs ciprofloxacin for the treatment of uncomplicated cystitis in women: a randomized trial. JAMA. 2005;23(8):949-955. doi:10.1001/ jama.293.8.949

20. Knottnerus BJ, Grigoryan L, Geerlings SE, et al. Comparative effectiveness of antibiotics for uncomplicated urinary tract infections: network meta-analysis of randomized trials. Fam Pract. 2012;29 (6):659-670. doi:10.1093/fampra/cms029

21. Ahmed H, Farewell D, Francis NA, et al. Choice of empirical antibiotic therapy and adverse outcomes in older adults with suspected urinary tract infection: cohort study. Open Forum Infect. 2019;6(3): ofz039. doi:10.1093/ofid/ofz039 
22. Márió G, Marianna A, Andrea L, et al. Revival of older antibiotics for the therapy of urinary tract infections: old, but gold Part 1: antimicrobial susceptibility of extended-spectrum $\beta$-lactamase-producing and AmpC $\beta$-lactamase-producing Escherichia coli isolates. Rev Med Microbiol. 2021;32(1):51-56. doi:10.1097/MRM.0000000000000220

23. Gupta K, Hooton TM, Naber KG, et al. International clinical practice guidelines for the treatment of acute uncomplicated cystitis and pyelonephritis in women: a 2010 update by the Infectious Diseases Society of America and the European Society for Microbiology and Infectious Diseases. Clin Infect Dis. 2011;52(5):e103-e120. doi:10.1093/cid/ciq257

24. Sadler S, Holmes M, Ren S, et al. Cost-effectiveness of antibiotic treatment of uncomplicated urinary tract infection in women: a comparison of four antibiotics. BJGP Open. 2017;1(3): bjgpopen17X101097. doi:10.3399/bjgpopen17X101097
25. Perrault L, Dahan S, Iliza AC, LeLorier J, Zhanel GG. Costeffectiveness analysis of fosfomycin for treatment of uncomplicated urinary tract infections in Ontario. Can J Infect Dis Med Microbiol. 2017;2017:6362804. doi:10.1155/2017/6362804

26. McKinnell JA, Stollenwerk NS, Jung CW, et al. Nitrofurantoin compares favorably to recommended agents as empirical treatment of uncomplicated urinary tract infections in a decision and cost analysis. Mayo Clin Proc. 2011;86(6):480-488. doi:10.1093/fampra/ cms029

\section{Publish your work in this journal}

Therapeutics and Clinical Risk Management is an international, peerreviewed journal of clinical therapeutics and risk management, focusing on concise rapid reporting of clinical studies in all therapeutic areas, outcomes, safety, and programs for the effective, safe, and sustained use of medicines. This journal is indexed on PubMed Central, CAS,
EMBase, Scopus and the Elsevier Bibliographic databases. The manuscript management system is completely online and includes a very quick and fair peer-review system, which is all easy to use. Visit http://www.dovepress.com/testimonials.php to read real quotes from published authors. 